\title{
Modeling of PMU-Based Islanded Operation Controls for Power Distribution Networks using Modelica and OpenIPSL
}

\author{
Biswarup Mukherjee $^{1} \quad$ Luigi Vanfretti ${ }^{2}$ \\ ${ }^{1}$ Indian Institute of Technology Bombay, India, bi smuk . ece egmail . com \\ ${ }^{2}$ Electrical, Computer, and Systems Engineering, Rensselaer Polytechnic Institute (RPI), USA, vanfrl@ rpi . edu
}

\begin{abstract}
This paper describes the modeling of a frequency controller that can be applied when islanding occurs at a power distribution network with a single distributed generator. The controller function requires bus frequency measurements which, for design purposes, need to be derived (computed) during dynamic simulations. Therefore, this paper also proposes a simple new frequency computation technique that can be used during dynamic simulations. The paper also addresses a technique for stochastic modeling of load uncertainties in the time-domain using the Modelica Noise library's features. The performance of the islanded controller is evaluated under load uncertainties, different PMU (phasor measurement unit) reporting rates and communication latencies.

Keywords: frequency computation, islanded controller, random load variation, PMU, distribution network, synchrophasors, Modelica, OpenIPSL
\end{abstract}

\section{Introduction}

\subsection{Motivations}

Islanded operation in power systems is required when a part of the network consisting of both loads and generation is isolated from the rest of the power grid, and generators continue to energize that isolated network (Almas \&Vanfretti, 2016). Controlling the frequency in an islanded power system is a very challenging task after an islanding occurs because it requires at least one generator in the island to restore the power/frequency balance in the island while at the same time restoring its mechanical speed before being re-synchronized to the main grid (Taranto \& Assis, 2012). Alternatively, if there are enough available generators in the islanded network, the generators could be used to operate the islanded portion autonomously, which is commonly referred to as a "microgrid" (Lasseter, 2002).

Other than having enough generation capacity to match the load in the island, at least one of the generators would need to be equipped with an isochronous controller to restore the frequency of the island to normal operating frequencies. However, this would require prior knowledge on how the microgrid will be formed and to equip all potential generators both with the traditional droop function and the isochronous function; and to know when to de-activate it. This paper explores an alternative supplementary controller that could provide the same functionalities and demonstrates this concept in the simplest case, when there is only one generator present in the island.

\subsection{Literature Review}

It is reported in (Franc, Taranto, \& Giusto, 2013) that synchrophasor-based islanding detection schemes may be able to provide fast and reliable islanding detection. To measure the frequency PMUs are proposed in (Kirkham et al, 2014), and controls for resynchronization using the PMU/phasor data have been studied in (Taranto \& Assis, 2012). For islanded operation, alternatively it would be equally attractive to propose a controller capable of using the frequency estimated by PMUs. This paper proposes to use PMU measurements in both transmission and distribution networks to achieve similar goals.

For simulation, authors in (Milano, 2017) have proposed that the system frequency can be estimated from the center of inertia (COI) concept and a washout filter (WF) on the phase angle of bus voltage. The COI is an artificial modeling construct and in practice it cannot be used (Diez-Marotoet al, 2001). In this paper, an alternative new frequency computation technique is proposed.

A synchronous islanding control scheme is proposed in (Jacobsen et al, 2016). It uses a load sharing concept, frequency deviation and phase angle deviation from the islanded network. The measurements from a PMU are used to calculate the active power imbalance with respect to the main grid's frequency and the phase angle. However, correction in the frequency's DC bias in the islanded network was not addressed.

For islanded operation, different governor configurations for frequency control have been proposed (Mahatet al, 2009). Such an approach requires an additional isochronous controller to bring 
the frequency back to its nominal value when the system is islanded. Instead, this paper proposes an alternative supplementary controller that is cascaded to the speed control loop.

\subsection{Contributions}

The main contributions of this paper are as follows:

- Proposing a simple frequency computation technique that uses bus voltage angles within the simulated model, which is attractive for controller simulations when using the positive-sequence power systems dynamic modeling framework.

- A new supplementary islanded operation controller is proposed. The controller uses a PI function and it is modeled using a centralized control architecture that receives data from PMUs, thereby complementing existing generator control systems instead of replacing the existing ones. When activated it will retain a frequency deviation of zero when the distribution side is islanded from the main transmission grid.

- A technique to simulate random load variations using Modelica Noise library ${ }^{1}$ (which is also integrated in Modelica Standard Library (MSL) $3.2 .2^{2}$ ) features is proposed. The performance of the islanded controller is evaluated under timedomain load uncertainties in the distribution side of the test network.

- The performance of the proposed controller is studied considering different PMU reporting rates and data transmission delays.

The remainder of this paper is organized as follows. In Section 2 the proposed frequency computation technique is presented. Sections 3, 4 and 5 explain the modeling of the islanded operation controller, stochastic load model, and a model to implement the PMU reporting rate with data transmission delay, respectively. Section 6 describes the power system and simulation execution models. Finally, case studies are analyzed in Section 7 and conclusions are drawn in Section 8 .

\section{Frequency Computation}

\subsection{Theory}

When the distribution grid is disconnected from the bulk transmission system, the bus voltage angles measured by PMUs in the distribution grid will deviate from those of the transmission grid. Phasor angle measurements are bounded to +/- 180 degrees. Therefore, if the bus frequency is calculated from the bus angle directly, angular measurement unwrapping will create spikes that corrupt the actual frequency

${ }^{1}$ Online at: https://github.com/DLR-SR/Noise

${ }^{2}$ Online at: https://github.com/modelica/ModelicaStandardLibrary deviation. To overcome this issue and to provide useful frequency signals for control a simple method is proposed. Let $V_{i}$ and $V_{r}$ represent the imaginary and real parts of complex bus voltage, then the bus angle $(\theta)$ can be calculated from these two values as

$$
\theta=\tan ^{-1} \frac{V_{i}}{V_{r}}
$$

Let $\omega$ be frequency of the bus voltage, then the first order derivative of the bus angle represents the frequency deviation at the bus. Therefore, the bus frequency can be represented as,

$$
\omega=\dot{\theta}=\frac{V_{r} \dot{V}_{i}+V_{i} \dot{V}_{r}}{V_{r}^{2}+V_{i}^{2}}
$$

Equation (2) is used for implementation in Modelica.

\subsection{Implementation}

Figure1 shows the block diagram used for implementation of the Modelica model of the proposed frequency computation technique.

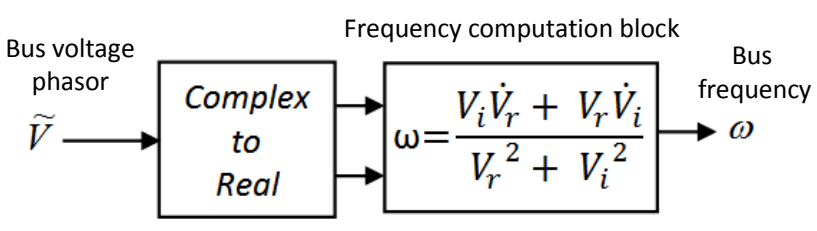

Figure 1. Block diagram for implementation of the frequency computation technique.

To compute the bus frequency the following Modelica code has been used in the "Frequency computation block" shown in Figure 1.

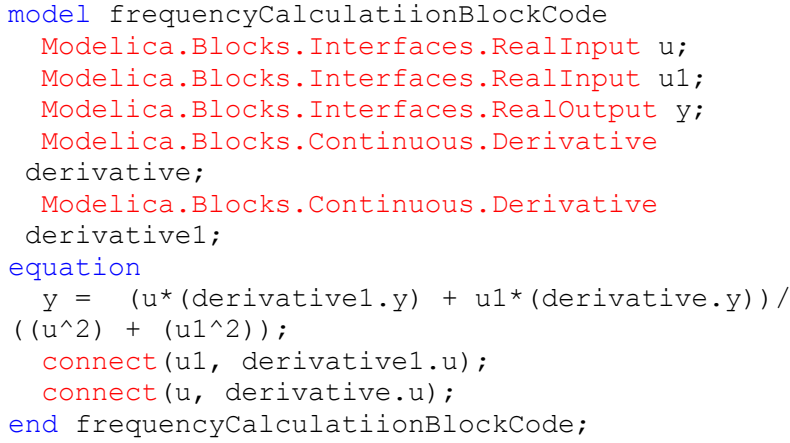

The real inputs $\mathrm{V}_{-} \mathrm{r}_{\text {and }} \mathrm{V}_{-} i$ represent the real and imaginary parts of the complex bus voltage and the real output $y$ represents the calculated bus frequency.

The Modelica code uses a derivative block from Modelica Standard Library (MSL), which is shown below. This block defines a transfer function between the input $u$ and output $y$. In the frequency computation 
the derivative block uses a gain value of $k=1$ and a time constant of $\mathrm{T}=0.01 \mathrm{sec}$.

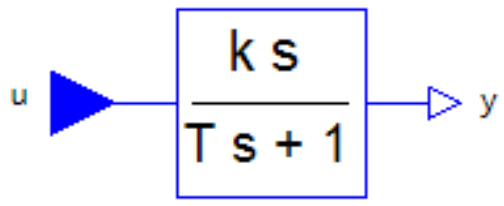

Figure 2. Model of the derivative block from the Modelica Standard Library (MSL).

\subsection{Numerical results and comparison}

Conventional power system tools, e.g. PSS/E (Siemens $\mathrm{AG}, 2018$ ), compute bus frequencies using the approach shown in Figure 3. It passes the bus voltage angle through a derivative computation and a first order filter. It has been shown in (Milano, 2017) that the approach is prone to numerical problems, although it is the standard de-facto approach. An alternative method in (Milano, 2017), is only suitable for domain specific power system tools and not general-purpose ones, e.g. Dymola or OpenModelica.

To illustrate the need for the implementation proposed in this paper, the standard approach, i.e. wash-out (WF) filter, is compared with the one proposed in this paper. The Modelica implementation of the WF filter is shown in Figure 3, while simulation results are plotted in Figure 4. The parameter used for the WF filter are $\mathrm{k}=1, \mathrm{~T}_{-} \mathrm{f}=1 \mathrm{sec}$ and $\mathrm{T}_{-} \mathrm{w}=2 \mathrm{sec}$, where $\mathrm{T}_{\_} \mathrm{f}$ and $\mathrm{T}_{-} \mathrm{W}$ are the time constants of the derivative and first order filter blocks, respectively. The gain $\mathrm{k}$ is the same for both derivative block and first order filter. In the simulation the input angle is switched from $-\boldsymbol{\pi}$ to $+\boldsymbol{\pi}$ with a period of $1 \mathrm{sec}$. to mimic angle wrapping.

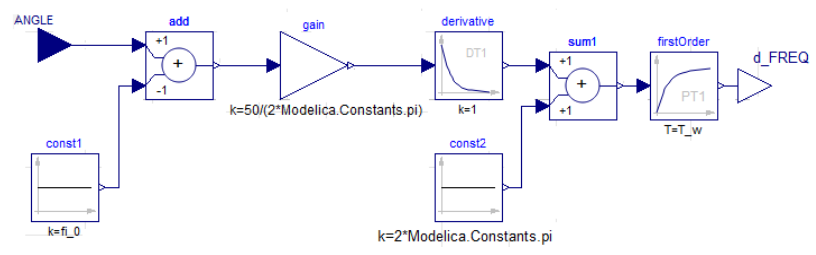

Figure 3. Washout filter (WF) implementation in Modelica.

The Figure $4 \mathrm{a}$ shows the traces of the proposed implementation showing $\Delta f=0$ for angle switching in Figure $4 \mathrm{~b}$ through $+/-\pi$ switching, while the blue trace shows the output of the WF filter. The expected output is a frequency value of $1 \mathrm{~Hz}$, consequently, two major issues with the WF filter can be observed: (1) the filter's response due to initialization and (2) numerical switching due to the sawtooth's input. Hence instead of using the traditional frequency computation approach, the islanded controller in the next section will use the proposed method for frequency computation.

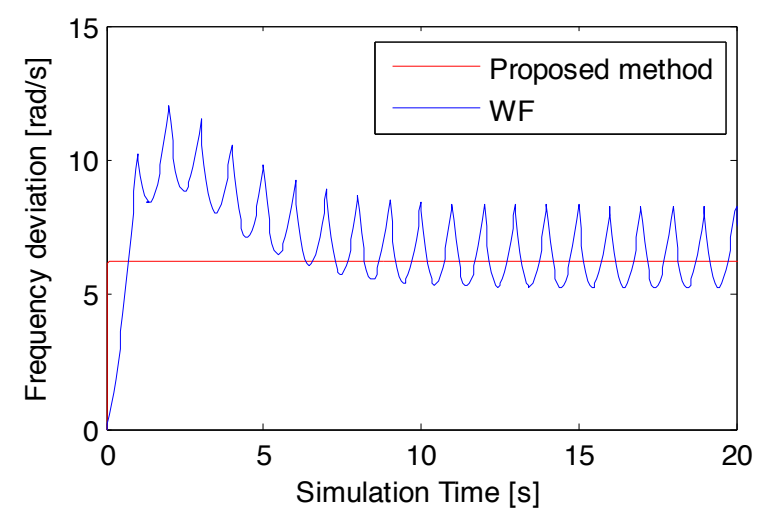

(a) Proposed method vs WF model

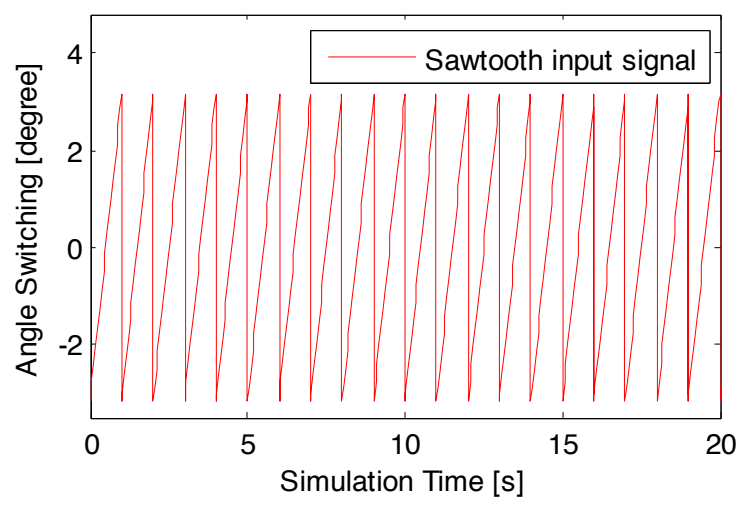

(b) Input signal

Figure 4. Comparison between the proposed frequency computation technique and the WF model.

\section{The Islanded Operation Controller}

\subsection{Islanded operation control function}

A schematic of the proposed islanded controller model is shown in Figure 5.

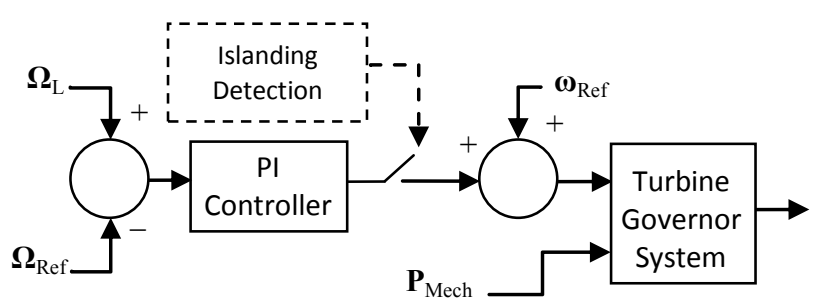

Figure 5. Schematic of the proposed controller.

The controller is activated when it detects that the distribution network is islanded from the transmission grid. In the schematic the error signal is obtained from the load bus frequency $\left(\boldsymbol{\Omega}_{\mathrm{L}}\right)$ and the reference frequency $\left(\boldsymbol{\Omega}_{\text {Ref }}\right)$. The output of this controller, along with the reference speed $\left(\boldsymbol{\omega}_{\text {Ref }}\right)$, provide the new input error signal of the speed control loop in the governor system. $\mathbf{P}_{\text {Mech }}$ represents the mechanical power input to the turbine corresponding to a prescribed power dispatch. 


\subsection{Modelica Implementation}

The Modelica implementation of the proposed controller is shown in Figure 6. The proposed controller is highlighted with a dotted line surrounding it, while the GENSAL block corresponds to the synchronous generator; IEEESGO corresponds to the gas and turbine model, and SEXS to the excitation control system of the generator. The overall system frequency is varied by introducing a speed change in the governor system of the transmission side generator model G1 in Figure 9 and 10.

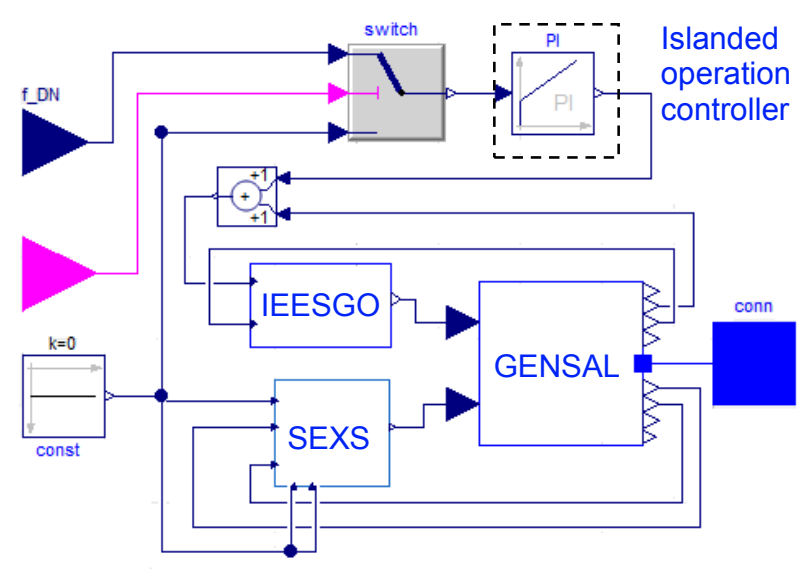

Figure 6. Modelica implementation for the distribution side generator model (G22) with the islanded operation controller, see Figure 9 for the network model.

The IEEE Standard for Interconnecting Distributed Resources with Electric Power Systems (IEEE Standard, 1547.2-2008) states that the DGs (Distributed Generations) must be disconnected from the isolated grid within $2 \mathrm{~s}$ after an unintentional islanding event. However, the goal of the proposed controller is to avoid DG disconnection and operate the grid autonomously. Hence, instead of tripping the generator, when the distribution side frequency reaches tripping thresholds, the trip signal goes to the breaker to island the distribution side, and the same time an activation signal 'start_islanding' activates the islanded operation controller. The PI controller's output can be expressed as in the Table 1 , where $K_{P}$ and $K_{I}$ represent the proportional and integral gain of the islanded operation controller respectively.

Table 1. Output truth table of voltage controller

\begin{tabular}{|c|c|}
\hline $\begin{array}{c}\text { Boolean signal } \\
\text { (start_islanding) }\end{array}$ & $\begin{array}{c}\text { Output of islanded } \\
\text { controller }(y)\end{array}$ \\
\hline True & $y=\Omega_{L} *\left(K_{P}+\frac{K_{I}}{S}\right)$ \\
\hline False & 0 \\
\hline
\end{tabular}

\section{Stochastic Load Modeling}

The Modelica Noise Library allows users to model stochastic behavior, and it can be used along with the load model with external input of OpenIPSL (Baudette et al, 2018) under, OpenIPSL.Electrical.Loads.PSSE.Load_E xtInput, to model the load uncertainties in any power network. Here, a white noise has been injected to the load model. Note that white noise generates a signal having normal distribution characterized by a mean and variance.

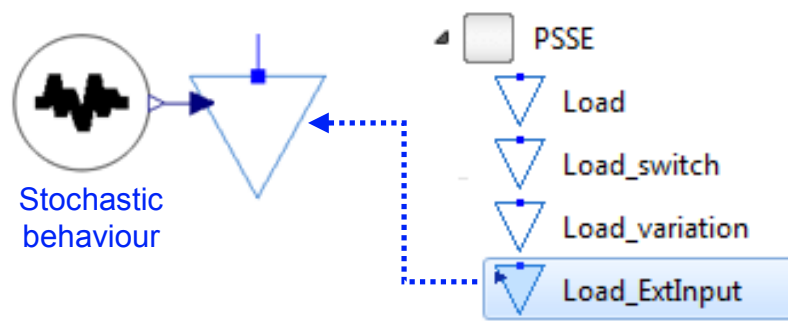

Figure 7. Stochastic model to simulate load variation in a power network.

\section{Modeling of PMU Reporting Rates and End-to-End Delay}

To Model the reporting rate of a PMU device, a Zero Order Hold (ZOH) block from Modelica Standard Library can be used to simulate different data "resolutions", i.e. different reporting rates, streamed by a PMU device. The time delay due to data transmission from a PMU to Phasor Data Concentrator (PDC) has been modeled using the 'fixedDelay' block from the MSL that uses the following Modelica code shown below. Note that it uses the Modelica 'delay' operator, which is a unique Modelica language feature. This operator introduces a fixed time delay between a real input and a real output signal.

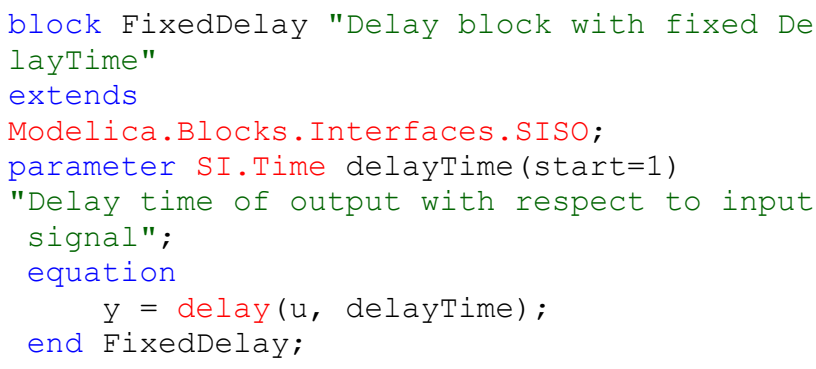

The implementation of the reporting rate and delay block is shown below, and the PMU reporting rate is shown in Figure 8. In this paper the performance of the controller has been studied for both the PMU reporting rate and delay due to data transmission by varying the sampling period of the $\mathrm{ZOH}$ block and the delay time of the 'fixedDelay' block. 


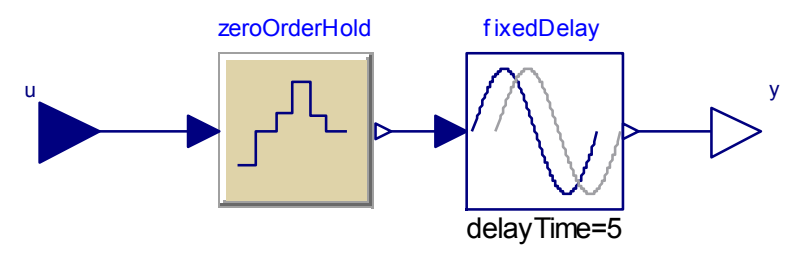

Figure 8. Modeling of PMU reporting rate and data transmission delay.

\section{Power System and Simulation Execution Models}

\subsection{Power System Model}

Figure 9 shows the power system model used for analysis. It is comprised by a transmission network and a distribution network. The circuit breakers CB1 and CB2 are controlled using logic equations implemented in a simulation set-up block, which is discussed next.

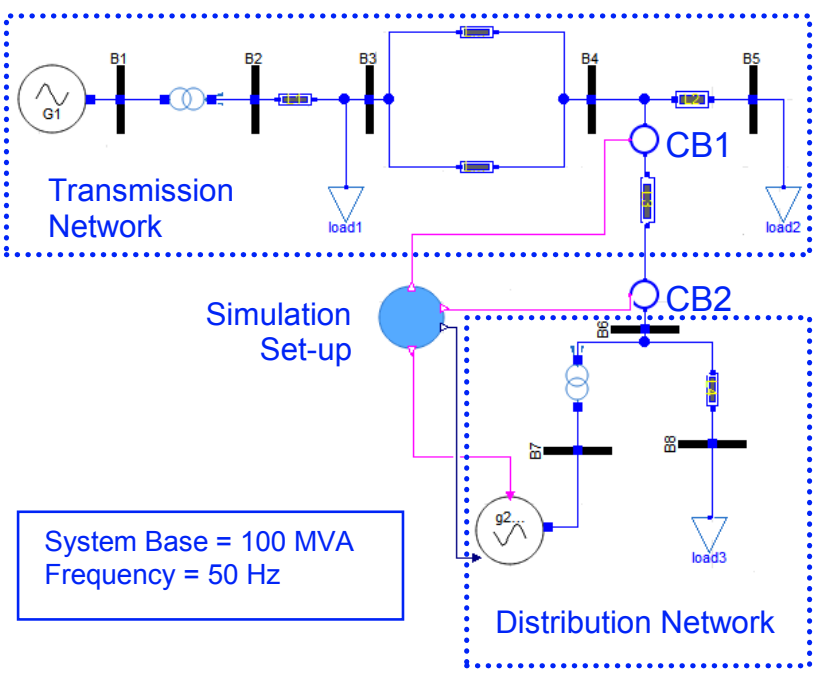

Figure 9. Modelica model of the test power system.

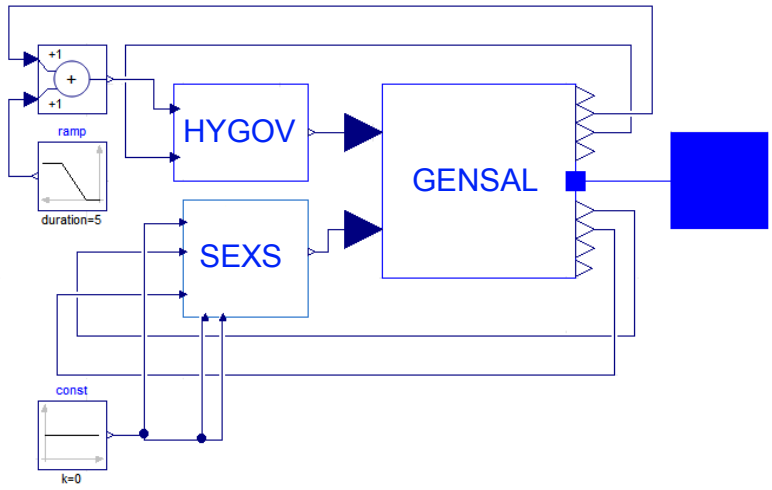

Figure 10. Generator model G1 in the transmission portion of the network.

\subsection{Simulation Set-up Implementation}

The implementation of the simulation set-up block is shown in Figure 11. It is used to create the islanding event and to activate the islanded operation controller. A ramp signal is activated in G1at $t=6$ seconds when the simulation starts and lasts for 5 seconds to vary the frequency. The 'Frequency computation block' calculates the frequency for the distribution side network from B6's bus voltage.

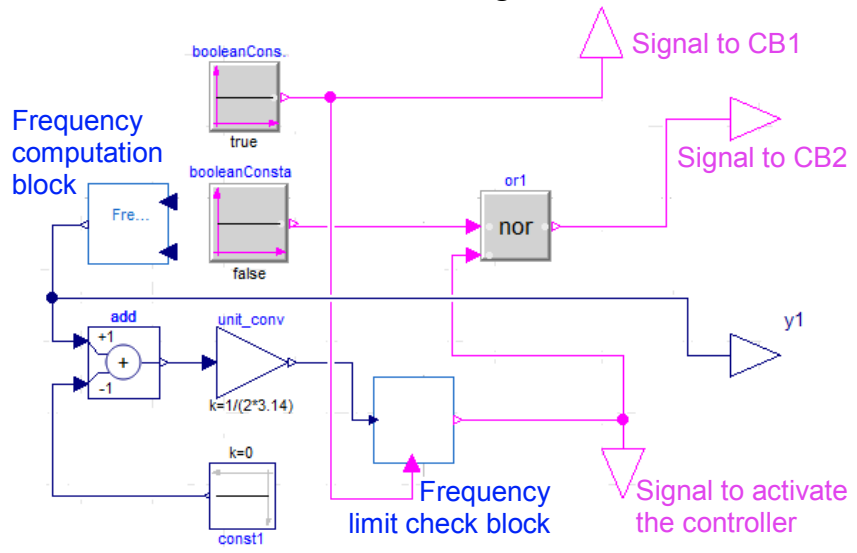

Figure 11. Modelica model of the simulation set-up block.

A true Boolean signal is sent to the circuit breaker CB2 when the frequency limit is reached. This condition checks the frequency deviation to the set-point limit set in the 'Frequency limit check' block. The output of this block is also used to activate the proposed islanded controller, while a Boolean true signal keeps the circuit breaker CB1 closed in the transmission side network while maintaining the transmission line energized. Regardless, CB1 is modeled in such way that it can be closed and open as CB2, which will be used in further studies. If the frequency limits are provided in nominal frequency values instead of a frequency difference, i.e. 49.95 and $50.05 \mathrm{~Hz}$ instead of $+/-0.05 \mathrm{~Hz}$, the block 'const 1 ' can be used to introduce a $50 \mathrm{~Hz}$ offset.

\section{Case Studies}

In the following case studies, except in Case 1, a steam turbine and governor system are used in the model of the distribution side generator G22 to analyze the performance of the proposed islanded operation controller. For all case studies, the same basic simulation set-up described in the previous section is used; hence, the disturbance applied corresponds to the ramp input into the governor reference as shown in Figure 10.

\subsection{Case 1:}

The feasibility of using proposed controller in either hydro or gas turbines is studied in this case. This is 
necessary as DER's include small hydro units, thermal and gas-power sources. In power systems, HYGOV is used to model hydraulic turbine and governor systems, while IEEESGO can be used to model steam turbine and governor systems.

The test system responses due to the controller's action were analyzed by plotting the frequency deviation in the distribution side network using both the HYGOV and the IEESGO turbine-governor systems. Figure 12 shows that the distribution side frequency deviation is zero when the proposed PI controller is activated, whereas a steady state error is present when there is no such control action regardless to the turbine-governor type.

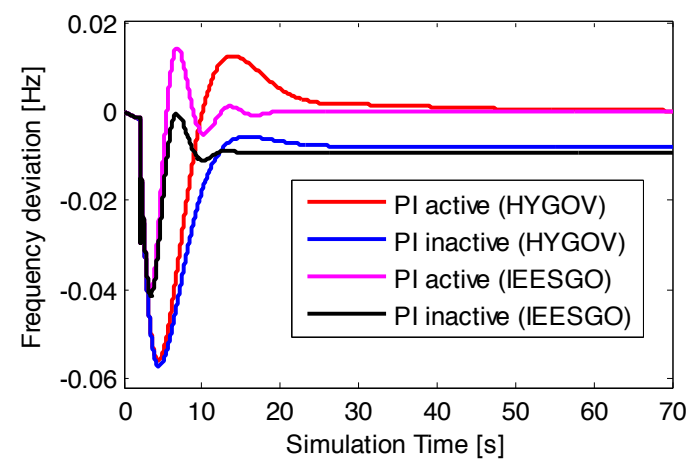

Figure 12. Case 1 - Frequency deviation for different turbine- governor systems.

In the case of the IEESGO turbine-governor system the maximum instantaneous values of frequency deviations are $0.0414 \mathrm{~Hz}$ and $0.0405 \mathrm{~Hz}$ respectively when the controller remains inactive and active. However In case of HYGOV turbine-governor systems the maximum instantaneous values are $0.057 \mathrm{~Hz}$ (when control action remains inactive) and $0.055 \mathrm{~Hz}$ (when control action remains active).

Observe that the responses of HYGOV results from a larger frequency transient which is due to the nonminimum phase characteristic of the hydro turbine. To minimize this transient or reduce it to allowable operational limits it is necessary to redesign the governor control system, which will be discussed in a future paper.

\subsection{Case 2:}

Here the controller's response has been analyzed by plotting the frequency deviation of the distribution network when the load in B6 has a noise level with standard deviation (s.d.) of 0.0001 . The results are plotted in Figure 13a. As it can be observed, the modeling of load uncertainties allows to evaluate the controller's effort during the islanding and also when normal operating conditions have been reached.

Figure $13 \mathrm{~b}$ helps to show the impact of stochastic loads. It shows the islanded operation control output error for both deterministic and stochastic responses. Note from Figure 13b that deterministic load models do not allow to accurately capture the controller's response due to time varying load changes. The ability to capture this behavior can allow to create controls that minimize the impact of stochastic variations on the turbine, which will be subjected to further work.

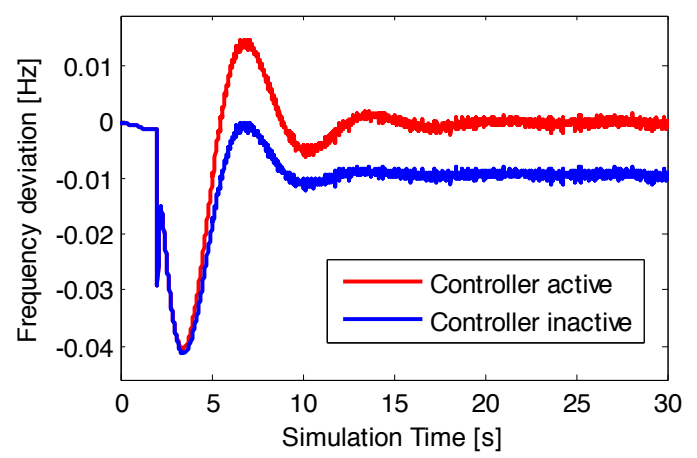

(a) Frequency deviation

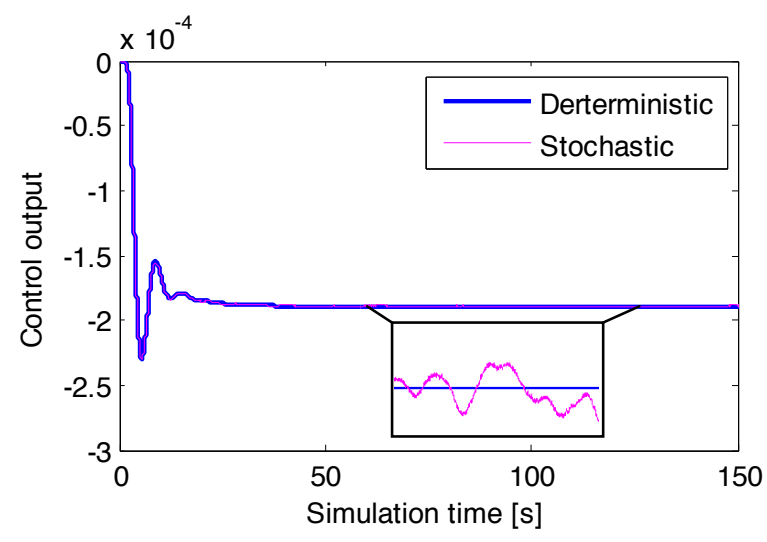

(b) Controller output

Figure 13. Case 2 - Stochastic and deterministic model responses.

\subsection{Case3:}

This case study has been carried out to analyze the performance of the islanded operation controller for the test network in Figure 9 considering the impact of the PMUs reporting rate. The frequency deviation has been plotted for different sampling periods $(\mathrm{ZOH})$ when the islanded controller is active. The plots for this case study are shown in the Figure 14a.

From Figure 14a observe that delays from 25 to 150 $\mathrm{ms}$ have no major impact on the controller's performance, this is because the frequency dynamics being controlled are much larger than typical PMU reporting rates. However as shown in Figure 14b, when the reporting rate is set to tens of seconds, the control loop becomes unstable i.e. for the reporting rate $>16 \mathrm{~s}$. This is a positive result, as typical PMU reporting rates are $\leq 16$ s, i.e. $10,30,50,60$ samples per seconds. 
Although the lowest PMU reporting rates is of 10 samples per second, simulations have been carried out with much slower reporting rates to determine the stability margin of the controlled system.

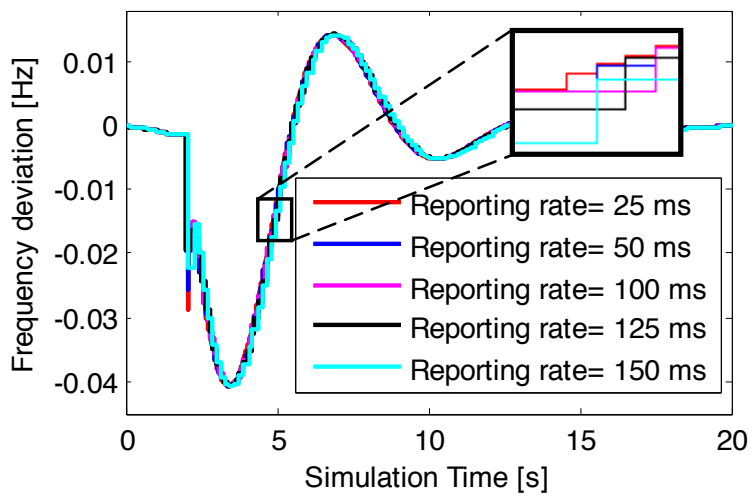

(a) Frequency deviation

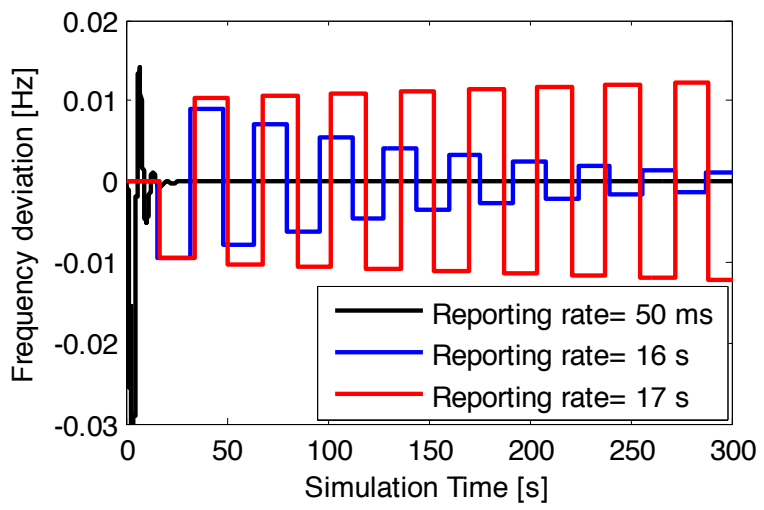

(b) Frequency deviation

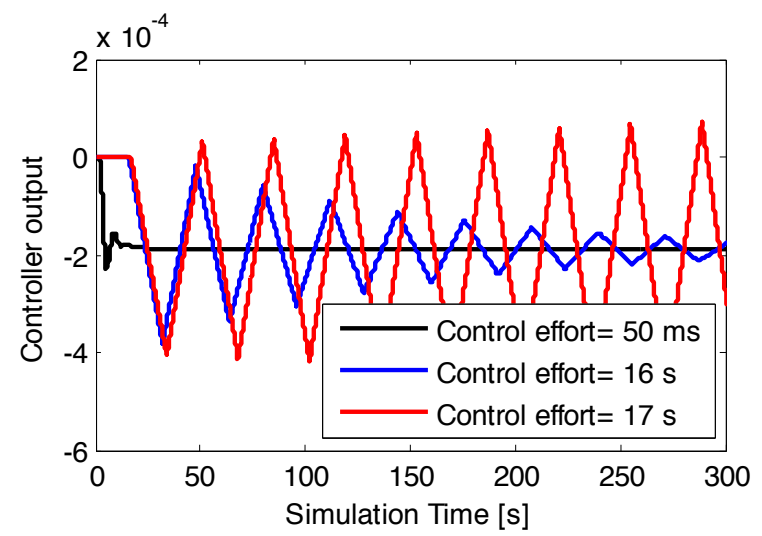

(c) Controller output

Figure 14. Case 3 - Analysis of different PMU reporting rates.

\subsection{Case 4:}

This case study analyses the impact of data transmission delay. A ' $\mathrm{fixedDelay}$ ' block is used to mimic the aggregate time-delay from a PMU device to the controller. The results are shown in Figure 15a and $15 b$.
As it can be observed in Figure 15a that the maximum delay bound is time delay $(\mathrm{td}) \approx 12 \mathrm{~s}$. These results are encouraging, as typical synchrophasor systems only incur in delays in the order of a 100s of milliseconds, up to a few seconds, and thus, delay compensation will not be critical as in other PMU based controls (Almas \&Vanfretti, 2016).

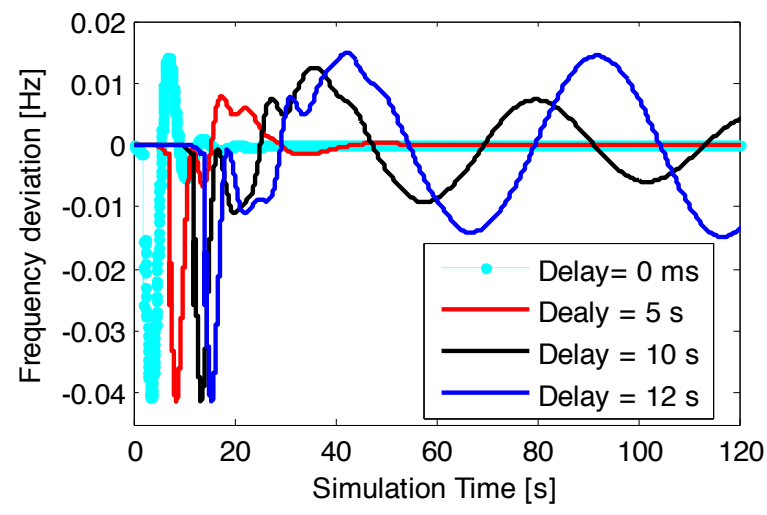

(a) Frequency deviation

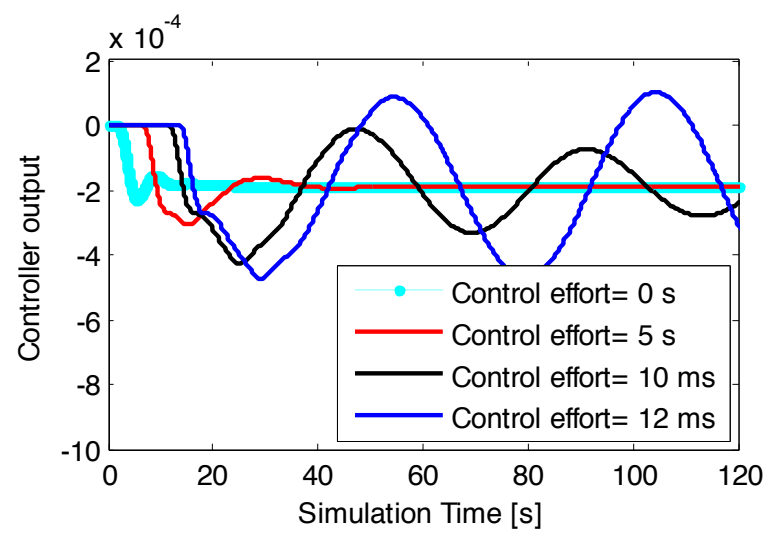

(b) Controller output

Figure 15. Case 4 - Controller output for different delays.

\subsection{Case 5:}

This case study analyses the impact of frequency deviation over both control error and mechanical power output of the turbine governor system. From Figure $16 \mathrm{a}$ it can be observed that for both deterministic load and stochastic load model the control error decreases up-to $0.023 \%$. This shows the impact of modeling the stochastic behavior of the load for control design. Meanwhile, Figure 16b shows the plot of frequency deviation for mechanical power output of the turbine governor system for both stochastic and deterministic load models. Observe that with the increase of the frequency deviation, the mechanical power increases up-to $10.88 \%$. This shows that stochastic load modeling is necessary when analyzing turbine-governor control systems. 


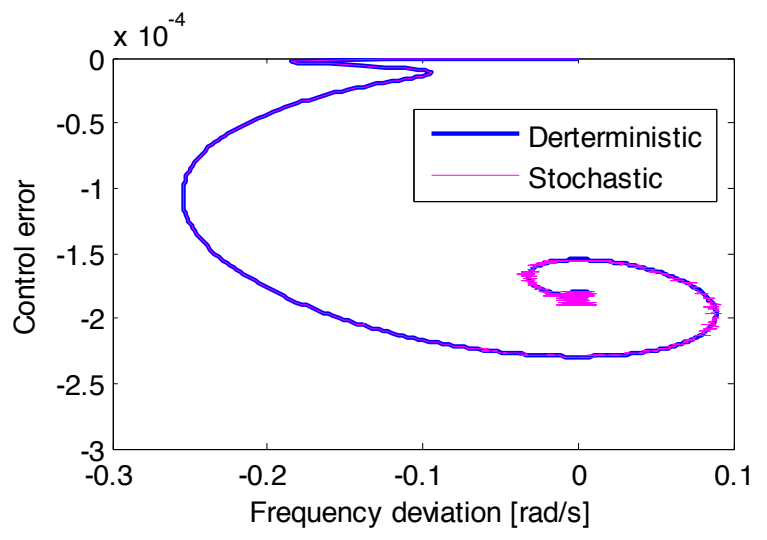

(a) Frequency deviation vs control error plot(see Figure $13 \mathrm{~b}$ for the time domain plot of the control error)

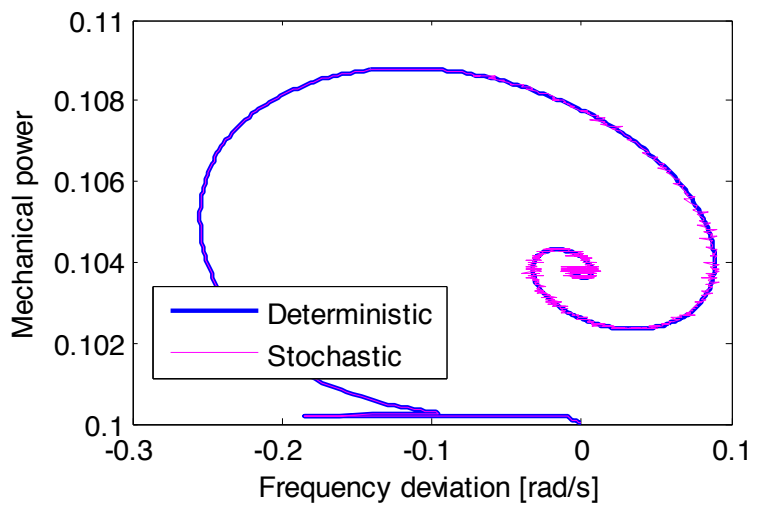

(b) Frequency deviation Vs mechanical power

Figure 16. Case 5 - Impact of stochastic load modeling in turbine-governor system control performance.

\section{Conclusions}

The following conclusions can be drawn from the work presented in this paper:

- The proposed frequency computation provides better results than the traditional WF filter in case of angle wrapping from $+/-180$ degrees.

- To simulate random load variations in any power network the Modelica Noise Library features is combined with OpenIPSL is a feasible solution that helps to capture the impact of time domain load/ generation uncertainties in the controller performance.

- The proposed supplementary islanded frequency controller can be activated to retain a frequency deviation of zero when islanding occurs. It also performs satisfactorily to correct the frequency deviations when subjected to load uncertainties.

- The current PMU report rates and typical delays that synchrophasor systems experience will not have a major impact on the controller performance.

Further work will be to investigate the performance of this controller under multiple realizations of load uncertainties (noise level) in order to analyze the impact of uncertainties on the system.

\section{Reproducibility of Research}

The models used to obtain the results in this paper are available online on the following Github repository: https://github.com/ALSETLab/2018_AmericanModeli caConf PMUBasedIslanding

\section{Acknowledgements}

The authors would like to acknowledge former members of the now extinct SmarTS Lab research group for their valuable feedback and comments towards building the models for this work, especially Tin Rabuzin.

Author B. Mukherjee was supported by Explora'Sup grant (Auvergne Rhône Alpes Scholarship) from Grenoble Institute of Technology (Grenoble-INP), for the year of 2016/2017.

The work of L. Vanfretti was also supported in part by the Engineering Research Center Program of the National Science Foundation and the Department of Energy under Award EEC-1041877 and in part by the CURENT Industry Partnership Program.

\section{References}

Almas, M.S. \&Vanfretti, L., "RT-HIL Implementation pf the Hybrid Synchrophasor and GOOSE-Based Passive Islanding Schemes," IEEE Transactions on Power Delivery, vol. 31, pp. 1299-1309, June 2016.

Taranto, G.N., Assis, Tatiana M.L. "Automatic Reconnection From Intentional Islanding Based on Remote Sensing of Voltage and Frequency Signals," IEEE Transsactions on Smart Grid, December 2012, Vol. 3, pp. $1877-1884$

Lasseter, R.H., "MicroGrids," Power Engineering Society Winter Meeting, 2002. IEEE, vol.1, no., pp.305,308 vol.1, 2002

Franc, R., Sena, C., Taranto, G.N., \& Giusto, A., "Using synchrophasors for controlled islanding-A prospective application for the Uruguayan power system," IEEE Trans. Power Syst., vol. 28, no. 2, pp. 2016-2024, May 2013

Kirkham, H., Dagle, J., Sun, Y., PMU Measurement Technology. s.1.: https://certs.lbl.gov/sites/all/files/kirkham-pmumeasurement-technology.pdf, 2014. p. 10

Milano, F., and Ortega, A., "Frequency divider," Power \& Energy Society General Meeting, 2017 IEEE

Diez-Maroto. L., Vanfretti. L., Almas, M.S., Jonsdottir. G.M., Rouco.L, “A WACS exploiting generator Excitation Boosters for power system transient stability enhancement," Elsevier-Electric Power Systems Research. https://doi.org/10.1016/j.epsr.2017.03.019

Jacobsen, M.R., Laverty, D., Best, R. J., “A laboratory experiment of single machine synchronous islanding using PMUs and Raspberry Pi - A platform for multi-machine islanding," Power \& Energy Society General Meeting, 2016 
PukarMahat, Zhe Chen, and BirgitteBak-Jensen, "Gas turbine control for islanding operation of distribution systems," Power \& Energy Society General Meeting, 2009 IEEE

Modelica Noise. [Online] https://github.com/DLR-SR/Noise

Modelica Standard Library, [Online] https://github.com/modelica/ModelicaStandardLibrary

Siemens AG. (2018). PSS $\AA$ E - high-performance transmission planning and analysis software. Retrieved from:

https://www.siemens.com/global/en/home/products/energy /services/transmission-distribution-smart-grid/consultingand-planning/pss-software/pss-e.html

IEEE Application Guide for IEEE Standard for Interconnecting Distributed Resources With Electric Power Systems, IEEE Std. 1547.2-2008, 2008

Baudette, M., Castro, M., Rabuzin, T., Lavenius, J., Bogodorova, T., \&Vanfretti, L. (2018). OpenIPSL: OpenInstance Power System Library - Update 1.5 to "iTesla Power Systems Library (iPSL): A Modelica library for phasor time-domain simulations", SoftwareX. https://doi.org/10.1016/j.softx.2018.01.002

OpenIPSL GitHub, [online] https://github.com/OpenIPSL/OpenIPSL .

Modelica Documentation, [Online] https://build.openmodelica.org/Documentation/ModelicaR eference.Operators. $\% 27$ delay()\%27.html

Almas, M.S. \&Vanfretti, L., "Impact of time-synchronization signal loss on PMU-based WAMPAC applications,"IEEE Power and Energy Society General Meeting (PESGM),pp.1 - 5,2016 